\title{
Diacronie
}

Studi di Storia Contemporanea

$N^{\circ} 14,2$ | 2013

Processo penale, politica, opinione pubblica (secoli XVIII-XX)

\section{Pulizia, giustizia e potere}

Ruolo e significato del Terrore nell'esperienza staliniana

\section{Filippo Ferrara}

\section{(2) OpenEdition}

\section{Journals}

\section{Edizione digitale}

URL: http://journals.openedition.org/diacronie/203

DOI: 10.4000/diacronie.203

ISSN: 2038-0925

\section{Editore}

Association culturelle Diacronie

\section{Notizia bibliografica digitale}

Filippo Ferrara, «Pulizia, giustizia e potere », Diacronie [Online], № 14, 2 | 2013, documento 7, Messo online il 01 août 2013, consultato il 30 avril 2019. URL : http://journals.openedition.org/diacronie/203 ; DOI : 10.4000/diacronie.203 


\section{Diacronie}

\section{$7 /$}

\section{Pulizia, giustizia e potere}

\section{Ruolo e significato del Terrore nell'esperienza staliniana}

Filippo FERRARA*

Prendendo in esame il caso studio dell'Unione Sovietica stalinista si è cercato di evidenziare la stretta connessione tra repressione pubblica e purghe; il ruolo svolto dai grandi processi deve essere confrontato e messo in relazione con le operazioni portate avanti segretamente in URSS. Come ha affermato Nicolas Werth, è necessario superare la consueta periodizzazione, che confina il Terrore al solo biennio 19371938: il Grande Terrore, fondamentale nell'edificazione della potenza sovietica, è da inserire in un più ampio arco cronologico, quello della dittatura di Stalin.

\section{Introduzione}

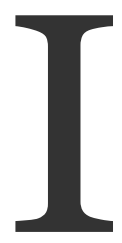

1 grande Terrore è stato il punto d'arrivo, radicale e micidiale, di una serie di operazioni repressive di massa, somiglianti a una vera e propria impresa d’ingegneria sociale, messa in atto dal regime staliniano tra la fine degli anni datata 1953. Sono necessarie due considerazioni preliminari: il grande Terrore, ebbe sì ufficialmente un inizio e una fine ben precisi, dal luglio 1937 al novembre 1938, ma va considerato "solo" come un tassello di un vero e proprio mosaico di morte architettato da Stalin negli anni della sua leadership. Non può essere considerato come un fenomeno a sé stante, limitandolo al biennio 1937-1938. Temporalmente va inquadrato, in primo luogo, come tappa successiva di una prima purga legata alla collettivizzazione delle campagne e all'industrializzazione forzata e che colpì in primis, tra il 1929 e il 1931, i kulaki e un gran numero di contadini che si rifiutarono di entrare nei kolchozy. 
In secondo luogo esso ha preceduto un'ondata repressiva, guidata dal presidente del Soviet Supremo Andrej Ždanov, che travolse l’intellighenzia appena concluso il conflitto. La seconda considerazione ci serve per evidenziare come il Terrore abbia viaggiato su binari tra loro al tempo stesso paralleli e intersecanti. Paralleli perché, come vedremo, alla facciata dei grandi processi di Mosca si affiancavano le operazioni segrete che andavano a colpire la cosiddetta "gente comune"; intersecanti perché senza i grandi processi non era possibile giustificare tali repressioni, e senza tali repressioni i grandi processi sarebbero stati quanto mai sterili. Esso fu quindi una macchina perfetta che trasformò nel giro di pochi anni quella che doveva essere la dittatura del proletariato prima in una dittatura del Partito sul proletariato e successivamente una dittatura del leader sul Partito, ripulendo lo stesso dagli oppositori, plasmando il proletariato ed eliminando quella parte di esso che veniva considerato per un qualsivoglia motivo sospetto, pericoloso.

\section{Il processo all'eresia trockijsta-zinovieviana}

Ponendosi su di presunta linea di continuità con Marx e con Lenin ${ }^{1}$, Stalin potè consolidare la sua figura come la reincarnazione del vero ed unico spirito comunista e mettere di conseguenza al di là, nelle sabbie mobili del revisiosismo, tutti coloro che ad esso - quindi a Lenin, quindi a Marx - si opponevano. Questa era solo la base ideologica che permise in qualche modo una giustificazione anche morale di quella che sarà poi la pratica, il Terrore. La sola propaganda ideologica, scatenata anche attraverso le quotidiane denunce della «Pravda», non era però sufficiente. Serviva un evento eccezionale per poter mostrare alla popolazione la reale esistenza di una frangia interna al Partito pronta a sovvertire lo Stato socialista, e conseguentemente dar via al ripulisti del Partito, tassello necessario per proseguire poi con lo sterminio delle opposizioni, o presunte tali, popolari. Tale evento fu l'omicidio il primo dicembre 1934 del dirigente bolscevico Sergej Kirov. Sulla sua morte è ancora aperto un dibattito. L'omicidio ufficialmente fu perpetrato per mano di un certo Leonid Nikolaev e organizzato dal cosiddetto "Centro trockijsta-zinov'eviano unificato", composto da quei sedici dirigenti del Partito (più Lev Trotsky e il figlio Sedov) che il 19 agosto 1936 si presentarono dinanzi al Tribunale militare di Mosca per rispondere a questa accusa e a quella per cui avrebbero gestito l'organizzazione di un centro terroristico nei cui programmi era l'uccisione dei massimi dirigenti del Partito. A partira dall'era Chruščëv si diffuse la

\footnotetext{
${ }^{1}$ Come emerge dagli estratti delle lezioni tenuti a Sverdlov, e raccolte poi nel Breve Corso.
} 
teoria, suggerita da Trotsky, che questo omicidio fosse stato ideato da Stalin stesso, anche perché durante il XVII Congresso del Partito, apertosi nel gennaio dello stesso anno, non solo Kirov fu l'unico a criticare ferocemente la linea stalinista, in particolar modo l'estorsione di cui erano vittime i contadini e gli abusi provocati dalle campagne di raccolta, ma raccolse il maggior numero di suffragi. Al di là di una qualsiasi interpretazione, questo caso servì a Stalin per imprimere un'accelerazione decisiva per eliminare una volta per tutte ogni forma di opposizione.

Il 24 agosto il presidente del Collegio Militare della Suprema Corte di Giustizia dell'URSS Vasilij Ulrich emise questa sentenza²:

[...] Con ciò fu accertato che: Zinov'ev Gregorij, Kamenev Lev, Evdokimov Grigorij, Bakaev Ivan, Mrac'kovskij Sergej, Ter-Vaganjan Vagarshak, Smirnov Ivan sono colpevoli : 1) di aver organizzato il "Centro terroristico trockijsta- zinov'eviano unificato" per assassinare i capi del governo sovietico e del partito comunista dell'URSS, 2) di aver preparato ed eseguito attraverso il gruppo terroristico illegale di Leningrado, Nikolaev- Kotolynov e altri, che il 29 dicembre 1934 furono condannati dal Collegio Militare della Suprema Corte di Giustizia dell'URSS, il delittuoso assassinio del compagno Sergej Kirov, 3) di aver organizzato una serie di gruppi terroristici che preparavano l'uccisione dei compagni Stalin, Voroscilov, Ždanov, Kaganovic, Ordzhonikidze, Kosior e Postyscev, cioè dei crimini contro gli articoli 58/8 e 58/11 del Codice Penale della RSFSR. Dreister Efim, Reingold Isaac, Pikel Richard, Gol'tsman Eduard, Fritz David, Olberg Valentin, Berman- Jurin Konon, Lurie Moisej, Lurie Nathan quali membri dell'organizzazione controrivoluzionaria terroristica trockijsta-zinov'eviana illegale di essere stati attivi partecipanti alla preparazione dell'uccisione dei capi del Partito e del Governo, dei compagni Stalin, Voroscilov, Ždanov, Kaganovic, Ordzhonikidze, Kosior e Postyscev, cioè dei delitti contro gli articoli 19 e 58/8, 58/11 del Codice Penale della RSFSR. Sulla base di quanto sopra esposto e in conformità con gli articoli 319 e 320 dell'ordinamento di procedura penale della RSFSR, il Collegio Militare della Suprema Corte di Giustizia dell'URSS condanna tutti e sedici gli imputati alla pena capitale mediante fucilazione e alla confisca di tutti i loro beni personali. Trockij Lev, attualmente all'estero, e suo figlio Sedov Lev, i quali attraverso le deposizioni degli imputati Smirnov, Gol'tsman, Dreister, Olberg, Fritz e Berman-Jurin, così come attraverso il materiale dell'attuale processo furono dichiarati colpevoli della preparazione diretta e della guida personale nell'organizzazione di atti terroristici nell'Unione Sovietica contro i capi del Partito comunista dell'URSS e dello Stato sovietico sono, nel caso in cui vengano trovati nel territorio dell'URSS,

\footnotetext{
2 Di cui viene riportata solo la parte conclusiva.
} 
immediatamente da arrestare e da consegnare al Tribunale del Collegio Militare della Suprema Corte di Giustizia dell'URSS 3 .

Dal punto di vista prettamente formale, giuridico, questa sentenza altro non faceva che applicare delle norme scritte, ufficialmente violate dai sedici imputati. La questione importante però è un'altra. Questi sedici imputati veramente avevano violato l'articolo 58 del codice penale della RFSFR, che puniva le attività controrivoluzionarie con la pena capitale? Davvero avevano progettato di eliminare fisicamente i più alti dirigenti del Partito, Stalin su tutti? Davvero avevano ucciso Kirov? Gli interrogatori compiuti dal procuratore Andrej Vyšinskij possono in tal senso aiutarci. Prendiamo ad esempio un estratto dell'interrogatorio di Lev Kamenev del 20 di agosto.

Vyscinskij: «Come giudica gli articoli e le dichiarazioni da lei scritti nel 1933, nei quali professa devozione al Partito? Menzogna?», Kamenev: «No. Peggio della menzogna.», V.: «Perfidia?», K.: «Peggio.», V.: «Peggio della menzogna, peggio della perfidia. Cerchiamo la parola. Tradimento?», K.: «Lei l'ha detta»4.

Il procuratore prosegue, incalzando nuovamente l'imputato in tal modo:

Vyscinskij: «Dunque alla lotta contro la direzione del partito e del governo siete stati spinti da bassi motivi, dalla sete di potere personale?», Kamenev: «Si», V.: «Non le sembra che in tutto ciò nulla vi sia di comune con gli ideali socialisti?», K.: «C'è la stessa rassomiglianza che corre tra rivoluzione e controrivoluzione», V.: «Quindi lei è dalla parte della controrivoluzione?», K.: «Si.», V.: «Di conseguenza lei si rende conto di aver perseguito la lotta contro il socialismo?», K.: «Ci rendiamo nettamente conto di aver condotto la lotta contro la direzione del partito e del governo che conducono il paese al socialismo», V.: «Per conseguenza lei è anche contro il socialismo?», K.: «Si»5.

Tradimento e controrivoluzione. Queste erano le due parole che tuonarono non solo nella Sala d'Ottobre, ove si svolgeva il processo, ma in tutta la Federazione. Il grande Kamenev, eroe rivoluzionario, aveva tramato, insieme ad altri quindici ex "fedelissimi", contro lo Stato, voleva ribaltarlo in nome dell'interesse personale e contro il socialismo stesso. Il nemico interno esisteva davvero allora, Stalin aveva ragione. O perlomeno questo si faceva intendere. Tutto era organizzato nel minimo dettaglio, le parole erano

3 Cit. in CONTESSI, Pier Luigi, I processi di Mosca. Le requisitorie di Vyscinskij, le accuse del Breve Corso e la denuncia di Khrushev, Bologna, Il Mulino, 1970, pp. 147-153.

4 Ibidem, p. 87.

5 Ibidem, p. 88. 
precise, "giuste", dovevano incastrarsi perfettamente nella sceneggiatura che andava poi a realizzare questo splendido film propagandistico. Anche il ruolo degli imputati doveva essere recitato alla perfezione. Le ultime affermazioni di questi dovevano portare l'esempio, o meglio l'immagine, del buon comunista che, disciplinatamente, rientrava nei ranghi dopo lunghi anni di smarrimento politico e ideologico, riconosceva la propria colpevolezza e quasi auspicava la condanna, come se volesse liberare l'anima da tanta infamia commessa.

Kamenev ad esempio dichiarò:

Io fui riaccettato nel Partito. Dopo tutti i miei errori mi furono affidati compiti e posti di responsabilità. Ora sono qui per la terza volta davanti al tribunale proletario sotto l'accusa di piani, propositi e azioni terroristiche. Due volte mi fu risparmiata la vita. Ma tutto ha un limite, anche la generosità del proletariato ha i suoi limiti e noi questi limiti li abbiamo toccati6.

Inoltre aggiunse:

Così noi servimmo il fascismo, così organizzammo la controrivoluzione nei confronti del socialismo, così preparammo e spianammo la via all'intervento armato delle guardie bianche. Questa era la nostra strada, questa è la fogna dell'infame tradimento e di ogni possibile sozzura in cui siamo sprofondati7.

Anche qui vediamo la scelta accurata dei termini da utilizzare: fascismo, controrivoluzione, “infame tradimento". A fargli da eco v'era Zinov'ev, il quale didascalicamente spiegò al meglio quanto il Politbjuro, quindi Stalin, voleva uscisse dal processo: «Il mio bolscevismo imperfetto si è tramutato in antibolscevismo; attraverso il trockijsmo sono pervenuto al fascismo. Il trockijsmo è una sottospecie del fascismo; lo zinov'evismo è una sottospecie del trockijsmo» ${ }^{8}$. Il messaggio era chiaro: chi si discostava dai dogmi del socialismo, ovvero quelli impartiti dal Politbjuro, quindi da Stalin, era contro. Ogni eresia, ogni dubbio, ogni imperfezione nel credo socialista diventava immediatamente antibolscevismo, quindi trotskismo, quindi fascismo, quindi un qualcosa da eliminare. È legittimo ipotizzare l'organizzazione a tavolino di domande e di risposte, ed è al tempo stesso necessario evidenziare il significato delle parole riportate: esse, attraversando quei veri e propri megafoni della propaganda stalinista in cui s'erano tramutati i rei confessi, giungevano in tutto il paese tracciando

\footnotetext{
${ }^{6}$ Ibidem, p. 144.

7 Ibidem.

8 Ibidem, p. 145.
} 
quella che doveva essere considerata la linea corretta, al di là della quale v'era l'eresia. Non vi è lo straccio di una prova, reale, legittimante l'accusa di Vyšinskij, anzi vi erano anche dei grossolani errori che in un processo normale, o perlomeno pseudo-legale, avrebbero smontato il capo d'accusa e salvato la pelle degli imputati ${ }^{9}$. E di quegli eventi che potevano divenire pretesto per un'accusa il procuratore si disinteressava. A Vyšinskij interessava poco avere tra le mani prove solide, tanto tutto era già deciso. Abbisognava di prove appariscenti, doveva sviluppare un'accusa spettacolare, non funzionale. Teatrale, non reale. A cosa potevano servire prove reali, ma "piatte", dinanzi a pirotecniche accuse? Cosa c'era di meglio se non un'ammissione pubblica di tradimento, filofascismo e controrivoluzione? Altra questione importante riguardava la difesa. Sarebbe stato un grave errore non garantirla ufficialmente; così, attraverso le formule della "rinuncia" e dell'autodifesa, si risolse la questione ${ }^{10}$. Non vi era alcun avvocato difensore e i rei confessi non avrebbero opposto alcuna linea difensiva, anzi avrebbero non solo descritto per filo e per segno il programma del Centro a cui erano affiliati, ma si sarebbero impegnati a collaborare con Vyšinskij, fornendo lui i nomi di altri terroristi interni al Partito, impegnati in attività controrivoluzionarie. Il procuratore potè così aprire immediatamente una nuova inchiesta, convocando a processo diciassette di loro.

\section{Il processo contro il Centro antisovietico trotskista e le purghe staliniane}

Il 23 gennaio 1937 ebbe inizio, sempre a Mosca, il secondo grande processo, contro il "Centro antisovietico trotskista".

Al centro del processo furono posti il sabotaggio, che andava a colpire ambiti economici, politici e militari, e la minaccia di un nemico esterno pronto - grazie alla collaborazione del nemico interno, di cui i 17 imputati erano la massima rappresentazione - a ribaltare il potere, a invadere l'URSS e a instaurare un regime di stampo capitalistico. E con la guerra di Spagna che, grazie all'invio di volontari italiani e tedeschi al fianco dei falangisti di Franco, assumeva sempre più il valore simbolico di opposizione fra i fascismi e gli antifascismi, quale pericolo più grave poteva essere indicato al popolo se non quello del sabotaggio per aiutare le nazioni ostili a invadere

\footnotetext{
9 Ad esempio una delle prove più forti di Vyšinskij era quella scaturita dalla confessione di Golt'sman, che affermava di aver incontrato Trotsky nel 1931 nella sua stanza di albergo, al Bristol di Copenaghen, hotel che in realtà non esisteva più da anni.

${ }^{10}$ Il diritto all'autodifesa era stato "garantito", ma rifiutato dagli imputati.
} 
l'URSS? Ecco perché, nonostante gli imputati non avessero il peso di quelli del processo dei sedici, il processo del 1937 assunse un’importanza strategica enorme. Anche qui gli imputati si tramutarono in megafoni del Politbjuro. Riportiamo a tal proposito un passaggio fondamentale dell'interrogatorio a Sokol'nikov nel quale si denuncia pubblicamente il ruolo di una categoria di soggetti che da lì a qualche mese, come vedremo a breve, verrà sterminata.

Vyscinskij: «In concreto, su quali forze contavate all'interno del paese? Sulla classe operaia?», Sokol'nikov: «No», V.: «Sui contadini dei kolchozy?», S.: «No», V.: «E su chi allora?», S. : «Francamente, bisogna dire che noi contavamo il poterci appoggiare sugli elementi della borghesia contadina», V.: «Sui kulaki?», S. : «Si»11.

Se il primo processo era servito a eliminare "vere" opposizioni, quindi anche importanti personalità, a condannare l'eresia trotskista-zinovieviana e a tacciare di tradimento antisovietico, di controrivoluzionario tutto ciò che non fosse allineato allo stalinismo, qui, attraverso dirigenti importanti, ma comunque di minor fama rispetto ai sedici, si condannavano varie forme di sabotaggio, tutte "personificate" durante la requisitoria di Vyšinskij. Individuato come Sokol'nikov rappresentasse gli interessi kulaki, il Procuratore ricordò il passato (e presente) trotskismo di altri imputati quali Nikolaj Muralov, Jakov Drobnis, Michail Bogulavskij, Jakov Livscitz e Leonid Serebrjakov. Karl Radek, per anni portavoce agli Esteri di Stalin, accusato di «aver tradito la patria e aver promesso ai nemici concessioni reali e territoriali», era, in considerazione del ruolo ricoperto, il miglior capro espiatorio possibile ${ }^{12}$; con lui, Jurij Pjatakov, che aveva avuto un ruolo di primissimo piano nell'industrializzazione del paese. Chi meglio di lui poteva giustificare le difficoltà incontrate nello sviluppo industriale? Ecco che bisognava dare un volto ai mali che minacciavano l'URSS. Ed è per questo che possiamo intendere il secondo processo di Mosca come un'opera propagandistica più puntuale e raffinata rispetto a quella svolta durante primo.

Il secondo processo di Mosca fu però solamente il primo terribile evento del 1937, l'anticamera di una serie di ondate repressive che da lì a poco investirono un'elevata parte della popolazione, il picco di un processo partito molti anni prima e legato alla collettivizzazione delle campagne. La purga delle campagne era infatti iniziata già nel 1929 e investì in particolar modo la "classe" dei kulaki. Ben lontana dall'essere l'operazione pianificata auspicata da Stalin, la dekulakizzazione si sviluppò in un

${ }^{11}$ CONTESSI, Pier Luigi, op. cit., p. 192.

12 Ibidem, cit., p. 195. 
processo caotico, incontrollato, risoltosi in una sconclusionato epilogo di deportazioneabbandono. Il soviet rurale deliberava l'arresto di un certo numero di contadini, o kulaki, i quali (centinaia di migliaia) venivano caricati su vagoni per il bestiame e abbandonati in baracche di fortuna o gettati nella steppa, senza alcun compito preciso, dove venivano uccisi da fame ed epidemie, il tutto senza passare attraverso alcun processo. Parallelamente, con lo stesso "metodo" era iniziata la caccia agli «elementi antisovietici», ovvero cittadini originari della Finlandia, dell’Ucraina, della Polonia e di altri paesi vicini: una vera e propria pulizia etnica basata sull'equivalenza «non sovietico» uguale «elemento politicamente sospetto e perciò da allontanare». La delicata situazione internazionale, con i venti di guerra che soffiavano sempre più insistenti, e la preoccupazione per il formarsi della temutissima quinta colonna, spinsero Stalin e il capo dell'Nkvd Nikolaj Ežov a modificare qualitativamente l'operazione repressiva. Non c'era più tempo: tutti i problemi dovevano essere risolti il più rapidamente possibile, e per farlo si rendeva necessario disporre di un'organizzazione puntuale, impeccabile. Nella primavera del 1937 l'Nkvd si mise in moto per raccogliere pseudo-documentazioni riportanti liste di soggetti «pericolosi». Alla fine di marzo Ezov fece recapitare a Stalin l'elenco delle categorie da colpire. Queste comprendevano: gli ex kulaki; i confinati «speciali» e gli esiliati di ogni genere; i byvsie, le «persone del passato» che annoveravano i membri delle élites economiche del vecchio regime (ex nobili, ex industriali, commercianti); gli ex funzionari del regime zarista; gli ex ufficiali e volontari dell'Armata bianca; coloro che avevano un tempo partecipato a insurrezioni contadine; i lisency, cioè individui privati dei diritti civili; i membri del clero e delle sette; coloro che erano stati membri di partiti politici avversari; gli individui esclusi dal Partito comunista per motivi politici; gli immigrati politici stabilitisi in URSS e gli emigrati rientrati in URSS; i cittadini sovietici che erano stati in contatto con rappresentanti diplomatici stranieri; i cittadini sovietici di origine polacca, tedesca, finlandese, lettone, lituana, che lavoravano in alcune «imprese strategiche»; gli individui ai quali era stata rifiutata la propiska, ovvero il permesso di residenza; i criminali recidivi13 ${ }^{13}$. Il 2 luglio 1937 scattava l' operazione kulak, che sancì l'inizio ufficiale del Grande Terrore. Stalin inviò la seguente direttiva segreta a tutti i dirigenti del partito a livello regionale e statale:

Il Comitato centrale propone a tutti i segretari di partito delle regioni e delle repubbliche, così come a tutti i responsabili regionali dell'Nkvd, di schedare tutti i

13 WERTH, Nicolas, Nemici del popolo. Autopsia di un assassinio di massa. Urss, 1937-1938, Il Mulino, Bologna 2011, pp. 59-61. 
kulaki e i criminali che hanno fatto ritorno, affinché quelli tra di loro che sono più ostili (prima categoria) possano essere immediatamente arrestati e fucilati per effetto di una procedura amministrativa semplificata davanti a una trojka; gli altri, quelli meno attivi (seconda categoria), ma nondimeno ostili, siano esiliati in regioni lontane del paese su ordine dell'Nkvd. Il Comitato centrale v'invita, entro giorni cinque, a proporgli la composizione delle trojki, il numero degli elementi da fucilare e di quelli da esiliare.

I. Stalin ${ }^{14}$

In pochi giorni a Mosca giunsero le risposte dei delegati, cosicché il 30 luglio Ežov firmò L'ordine operativo del commissario del popolo agli Affari interni dell'URSS n. oo447 sulloperazione di repressione degli ex kulaki, dei criminali e di altri elementi antisovietici. Il tutto doveva esser fatto nella maggior segretezza possibile ${ }^{15}$. Parallelamente all'operazione kulak, Stalin ed Ežov lanciarono una decina di altre operazioni di massa, definite operazioni nazionali. Le procedure erano differenti: se la gestione dell'operazione kulak era rimessa nelle mani delle varie trojki regionali e locali, per le operazioni nazionali il verdetto emesso dalle dvojki doveva essere ratificato a Mosca, da Ežov o Vyšinskij. Vennero creati degli album, contenenti tutte le condanne proposte. Quando però gli album divennero troppi e le carceri sovraffollate, si decise di abbandonare questa procedura e istituire delle trojki speciali in ogni regione sul modello di quelle che si occupavano dei kukaki. Questo fu il bilancio del biennio: nel 1937 la polizia politica arrestò ufficialmente 936.750 persone, condannandone 790.665, di cui 353.074 a morte; nel 1938 vi furono 638.509 arresti, 544.258 condanne di cui 328.618 a morte ${ }^{16}$. A queste vanno poi aggiunte le condanne illegali, figlie di torture o di fucilazioni sommarie ${ }^{17}$.

\section{Il terzo processo e la fase finale del Terrore}

Abbiamo già sottolineato come le due vie del Terrore viaggiassero parallele, come due purghe distinte ma intrecciate, una "bassa" che colpì la gente comune, e una "alta" che colpì le élites, atta a ripulire il Partito fino a fondo, dai dirigenti di primo piano fino

\footnotetext{
14 Ibidem.

15 Ibidem.

${ }_{16}$ Cit. in GRAZIOSI, Andrea, L'URSS di Lenin e Stalin. Storia dell'Unione Sovietica 1914- 1945, Bologna, Il Mulino, 2007, pp. 414-415.

17 La cui entità è variabile a seconda delle supposizioni. La forbice, comunque troppo ampia - si va dai 2 agli 11 milioni - non permette una quantificazione precisa e certa.
} 
ai piccoli emissari regionali ${ }^{18}$. Questa culminò nel marzo 1938 con il terzo processo pubblico di Mosca, mentre l'altra proseguì fino a novembre ${ }^{19}$. La condanna dei ventuno, fra cui spiccavano i nomi di Nikolaj Bucharin, Aleksej Rykov e Genrich Jagoda ${ }^{20}$, fu l'ultimo atto dell'epurazione ufficiale che sancì la definitiva e irreversibile vittoria della linea staliniana. Non c'era ormai in tutto il paese alcun cervello capace di rilanciare un'alternativa alla formulazione staliniana della teoria marxista- leninista. Il grande insegnamento storico di quest'ultimo processo consisteva infatti nella condanna dell'ultima, forse della più forte e reale, opposizione allo stalinismo, il bucharinismo ${ }^{21}$. La condanna di Bucharin, e della corrente che egli aveva da anni rappresentato, doveva legittimare davanti alla storia l'opera di Stalin, ponendolo definitivamente come il solo e unico legittimo erede di Lenin. La purga nel Partito era così completata. Anche in quest'ultima parodia giudiziaria, l'attore principale fu Andrej Vyšinskij con una requisitoria tanto perfetta quanto grottesca. Ovviamente tutti i mali dell'URSS furono addebitati ai ventuno accusati: «Organizzando il sabotaggio, Rykov e Bucharin, Jagoda e Grinko, Rosengol'ts e Cernov perseguivano uno scopo ben determinato: tentar di strangolare la rivoluzione socialista con l'ossuta mano della carestia»22. Quindi, stando all'accusa del Procuratore la terribile carestia del 1932, fu provocata da ventuno sabotatori e non da una politica economica del Politbjuro, tanto folle quanto brutale, o quantomeno incapace di risolvere i problemi enormi figli di un periodo durissimo per il popolo russo, che in pochi anni aveva vissuto una guerra mondiale, una rivoluzione e una guerra civile, e che veniva da una precedente e decennale politica economica zarista altrettanto folle e brutale. E ancora: «Sharangovic provoca l'anemia dei cavalli e muoiono 30.000 animali. In più semina coscientemente la discordia nazionale, sviluppando sentimenti nazionalisti tra le popolazioni della Bielorussia» ${ }^{23}$. Il picco di assurdità si raggiunse quando si millantò la tendenza di Rykov e Bucharin al terrorismo, al Terrore come mezzo per raggiungere il potere. Un'accusa quantomeno assurda fatta da chi, come abbiamo visto, utilizzò davvero il Terrore come mezzo per raggiungere e consolidare il potere assoluto. Una delle armi principali di Vyšinskij era il ribaltamento della realtà. Sugli accusati cadevano tutte le colpe, e addirittura tutti i

\footnotetext{
18 La cosiddetta purga delle élites riguardò piccoli dirigenti regionali. Come a Mosca, anch'essa fu pubblica, anche se logicamente ebbe meno risonanza. Ad essa si può affiancare anche la purga degli ufficiali, che colpì gran parte dei piani alti dell'Armata Rossa, in primis Tuchachevskij. Questa, però, essendo stata svolta nella più completa segretezza, non ha fonti totalmente attendibili; per questa ragione si è deciso di non trattarne direttamente.

19 GRAZIOSI, Andrea, op. cit., p. 417.

${ }^{20}$ Quest'ultimo predecessore di Ezov alla guida dell'Nkvd.

21 WERTH, Nicolas, Storia della Russia nel Novecento, p. 306.

22 Cit. in CONTESSI, Pier Luigi, op. cit., p. 354.

23 Cit. in Ibidem, p. 355.
} 
crimini, del governo. L'unica forma di Terrore attuata in URSS in quegli anni era quella impartita da Stalin e coordinata da Ežov. Terrore che, come detto, proseguì fino a che Stalin inviò, il 17 novembre, una risoluzione segreta a un numero ristretto di alti responsabili, dell'Nkvd e della procura, dove si abolivano tutte le giurisdizioni speciali costituite dall'agosto 1937, si bloccavano immediatamente tutte le operazioni di massa e si criticavano i «gravi errori» e le «deformazioni» nel lavoro degli organi dell’Nkvd. Questi «gravi errori» erano così spiegati: «nemici del popolo»e «spie al soldo dei servizi stranieri» erano riusciti a penetrare nell'Nkvd con l'obiettivo di sottrarre questa istituzione al controllo vigilante del partito e a infiltrarla al fine di svilupparvi le loro attività sovversive e il «lavoro sotterraneo antisovietico»24. Ma perché Stalin decise di colpo di fermare il Grande Terrore? Anzitutto perché, già dall'estate del 1938, si era reso conto che i pieni poteri concessi all'Nkvd avevano introdotto un pericoloso squilibrio nel funzionamento del sistema e potevano porre le condizioni per la creazione di una possibile opposizione. In più la conferenza di Monaco mostrò come la politica di sicurezza collettiva condotta dalla metà degli anni Trenta era miseramente fallita. Il mancato invito dell'URSS in quella che sarà l'ultima conferenza in periodo di pace, il patto di non aggressione tra Francia e Germania e il patto anti-Comintern, stipulato dallo stesso Reich col Giappone, sembravano concretizzare quell'«accerchiamento imperialista» che terrorizzava l'Unione Sovietica sin dai tempi di Lenin. Questa delicata situazione internazionale ebbe, senza dubbio alcuno, un peso determinante sulla decisione di Stalin di porre fine al Terrore e ai disordini da esso provocato, non solo nella popolazione, ma anche all'interno dello stesso $\mathrm{Nkvd}^{25}$.

Interessante, per comprendere il "modo" in cui avvenivano queste operazioni, è il racconto, fatto anni dopo, del capo della polizia della città di Saratov: «[...] Le istruzioni di base erano le seguenti: produrre il maggior numero di casi, il più rapidamente possibile, formulandoli nel modo più semplice possibile» ${ }^{26}$. La prima cosa che salta all'occhio è proprio quel produrre casi che fa comprendere la veridicità giuridica su cui si basavano tali operazioni. Il caso non andava discusso, aperto, magari scoperto: andava prodotto. Questo rientra perfettamente nell'invito a "fare numero", impartita dall'ottobre 1937. In poche parole, veniva prefissato un numero da raggiungere, una quota precisa di persone da arrestare, ed questo doveva essere raggiunto con qualsiasi mezzo. Il che era possibile solo producendo casi. La necessità di accelerare i ritmi, di

\footnotetext{
${ }^{24}$ Cit. in WERTH, Nicolas, op. cit., p. 199.

25 Questo fu l'ultimo settore purgato prima del conflitto. Ezov e altri numerosi dirigenti furono arrestati e condannati a morte.

${ }^{26}$ Cit. in WERTH, Nicolas, op. cit., p. 112.
} 
produrre "casi collettivi" 27 , complicava però il compito degli agenti dell'Nkvd, i quali trovarono subito la soluzione per razionalizzare il lavoro, inventando. L'arbitrarietà assoluta nelle indagini si evidenzia riportando la diretta testimonianza di un agente dell'Nkvd:

[...] mi assegnò un numero minimo di individui da arrestare. Quando gli feci notare che, nel corso dell'istruttoria, si rischiava di avere difficoltà ad alimentare l'accusa di spionaggio, mi rispose così: «Fate così: scrivete voi stessi il protocollo. Immaginate in relazione al luogo nel quale lavorava l'individuo, il tipo di spionaggio che poteva fare, oppure incriminatelo per atti di diversione. Suvvia, non abbiate paura di nulla, non andremo tanto per il sottile. Se l'individuo non vuole firmare il protocollo, picchiatelo fino a che firmi» ${ }^{28}$.

L'ultima frase non lascia spazio ad alcun tipo di interpretazione. L'unica prova che potevano fornire era la confessione, quindi la firma del protocollo, e per giungere ad essa non bisognava escludere nessun mezzo necessario, compresa la tortura.

\section{La zdanovscina e la persecuzione degli intellettuali}

La più grande testimonianza per quanto concerne i metodi di interrogatorio, e non solo, è senza dubbio quella contenuta nel «saggio d'inchiesta narrativa», scritto dal saggista, deportato nei Gulag nel 1946, Aleksandr Solzenicyn, vittima di quell'ultimo ramo del Terrore, gestito ideologicamente dal ministro Andrej Ždanov, e che è meglio conosciuto come zdanovscina. Si trattò di una vera e propria caccia alle streghe in salsa sovietica, condotta essenzialmente in ambito culturale, in quanto ad essere perseguitati furono artisti e letterati. Vinta la guerra, il timore di Stalin si riversò sullo stato mentale del ceto intellettuale sovietico, un ambiente ad alto rischio di libero pensiero. Le critiche vennero rivolte prima verso singole opere e personalità considerate diseducative e poco edificanti. Esteriormente essa si attuava secondo la parola d'ordine della democrazia, e ogni decisione veniva formalmente sottoposta al vaglio del pubblico. Il regime dava così al popolo l'illusione di partecipare alle grandi decisioni e al tempo stesso esercitava pressione sull'intellighenzia, che veniva così messa alla gogna dal pubblico. Varie centinaia di migliaia di intellettuali furono arrestati, deportati in Siberia e giustiziati. Il controllo ideologico, poi, travolse tutti i campi della

27 Comodi per un raggiungimento più rapido del numero prefissato.

${ }^{28}$ WERTH, Nicolas, op. cit., p. 137. 
conoscenza, poiché il partito legiferava in storia e in filosofia, in linguistica, biologia, matematica e condannò addirittura alcune scienze «borghesi», quali la meccanica ondulatoria, la cibernetica e la psicanalisi. Come testimoniano gli archivi del Gulag, quegli 8 anni, dal 1946 alla morte di Stalin, conobbero un forte aumento del numero dei detenuti nei campi di prigionia e nelle colonie di lavoro, oltre 2.500.00o, e del numero dei deportati “speciali”, circa 2.700.000²9. Fra questi vi era Aleksandr Solženicyn.

\section{Conclusioni}

L'istruttoria non veniva compiuta per accertare la posizione dell'imputato, la veridicità dell'accusa ma, attraverso l'ampio utilizzo della tortura, per consolidare la propria accusa, per affermare la propria verità. La prima ambiguità è prettamente tecnica. In un regime legale, si dà il via a un'istruttoria dopo aver compiuto un'indagine, fatta apposta per assicurarsi dell'esistenza di motivi validi per procedere ad essa. Qui nessun organo operò alcuna indagine. Agli elenchi di persone stilati da dirigenti dell'Nkvd, seguiva l'arresto e il tempo destinato all'istruttoria non serviva a indagare, bensì a torturare, stancare, distruggere psicologicamente e fisicamente l'accusato per portarlo alla confessione, che nella quasi totalità dei casi veniva rilasciata per interrompere tali sofferenze ${ }^{30}$. Vediamo allora quali procedimenti venivano utilizzati per stroncare la volontà e la personalità del detenuto. Vi erano due metodi, quelli psicologici e quelli fisici. I metodi psicologici agivano con forza su soggetti psicologicamente deboli, i quali per quanto sapessero di essere innocenti, non resistevano alla brutalità dell’intimidazione («Lei non confessa? Dovrà fare un giro alla Sovloki. Lasciamo libero chi confessa»), del gioco sull'affetto dei familiari («Arresteremo vostra figlia e la metteremo in una cella con delle sifilitiche»), delle menzogne (utilizzate per agire sui parenti o sugli amici con la formula: «Se voi non farete queste deposizioni ne soffrirà lui... Solo firmando questo documento lo potete salvare»; il documento prevedeva la confessione, per esempio, della madre dell'imputato, ritenuta fonte più che attendibile, cosicché la firma della stessa diveniva una prova inattaccabile, di fatto una condanna). Inevitabilmente questi finivano per confessare. Era normale interrogare in piena notte, così da avere un interrogato meno lucido, meno equilibrato, più malleabile. Vi era poi la persuasione: «Lo vedi da te, una pena la dovrai pur scontare. Ma se resisti perderai la salute qui in prigione, ci lascerai la

\footnotetext{
29 Ibidem, p. 416.

$3^{\circ}$ SOLZENICYN, Aleksandr, Arcipelago Gulag. Saggio d'inchiesta narrativa, vol. I, Milano, Mondadori, 2009, cit. p. 112.
} 
pelle. Firma subito, dammi retta»31. Il tutto veniva compiuto dopo aver umiliato preventivamente gli accusati, costretti, nell'attesa d'esser interrogati, a stare sdraiati nel corridoio per diverse ore col divieto di sollevar la testa o aprire bocca, con il semplice scopo di avere un interrogato moralmente distrutto, umiliato, depresso. Qualora non fosse bastato l'attacco alla mente, si passava al fisico. I metodi fisici erano svariati e difficilmente classificabili. I più semplici andavano dal mezzo sonoro (si faceva sedere l'arrestato a una distanza di sei-otto metri dal giudice e lo si costringeva a parlare forte, fino a sfinirlo, lasciandolo senza voce; oppure gli si urlava nelle orecchie con dei megafoni fino a renderlo sordo), allo spegnimento di sigarette sulla pelle del detenuto, fino al mezzo luminoso (una fortissima luce elettrica, veniva puntata sul prigioniero ventiquattr'ore su ventiquattro) ${ }^{32}$. Vi erano poi forme più elaborate, per esempio il viavai continuo tra cella e interrogatorio. Il prigioniero veniva chiamato, portato dinanzi al giudice, poi, senza ricevere domanda alcuna, riportato in cella, poi nuovamente dal giudice e così via, per dodici o ventiquattro ore consecutive. La cella era il primo luogo dove si compivano le torture. Esse contenevano i box, degli armadi dove venivano scaricati i detenuti, costretti a stare, murati vivi, per intere giornate, senza cibo, senza luce, senza aria e, alcune volte, in compagnia di migliaia di cimici o altri insetti. Quando non c'erano box liberi, veniva creata una fossa, profonda tre metri e larga due, all'aria aperta, dove venivano gettati i detenuti "in esubero", obbligati a star lì, sotto la pioggia, la neve, nel gelo per giorni interi. Altre volte agli imputati veniva intimato di scavarsi da soli fosse singole, delle misure di una tomba: questo colpiva non solo il fisico, ma anche, se non soprattutto, il morale. Alcuni detenuti venivano obbligati a stare in ginocchio per lunghissimo tempo, persino quarantotto ore; i più sfortunati tra questi si tramutavano in latrine per i giudici. Un buon mezzo era poi l'insonnia, che devastava l'individuo senza lasciare tracce apparenti sul fisico. Più fantasiose erano le violenze fisiche vere e proprie: si poteva incappare in macchinette per stringere le unghie, camicie di forza, pratiche di rottura della spina dorsale, cerchi di ferro per stringere il cranio, roventi mazze di ferro infilate nell'ano ( il cosiddetto "marchio segreto"), stivalate sui testicoli, violenze di gruppo sulle donne imputate, candele spente su qualsiasi punto del corpo, fino alle costole rotte e ai denti spezzati33. Vedere i segni e sentire i racconti delle torture dei compagni di sventura era un'altra forma molto forte di condizionamento. Accorgersi che l'accusato interrogato precedentemente non era, ad esempio, in grado di sedersi a causa delle natiche devastate dai manganelli,

\footnotetext{
${ }^{11}$ Ibidem, p. 118.

32 Ibidem, p. 123.

33 Ibidem, pp. 122-129.
} 
incideva sulle deposizioni. Questo portava a firmare e ad affidarsi alle proprie doti fisiche per sopravvivere ai lavori forzati, alla prigionia, alla fame, laddove l'opzione era quella di farsi devastare il corpo finché, giunti allo stremo delle forze, non fosse stato necessario per i prigionieri forzarsi a fornire a questi giudici-macellai, come li chiamò Solzenicyn, la confessione, pur di mantenersi in vita. Uno dei principi essenziali dell'istruttoria era poi quello di privare l'imputato della conoscenza delle leggi. Quasi sempre l'interrogatorio iniziava in tal modo: «Lei è accusato secondo gli articoli 58-10 parte 2 e 58-11 del codice penale della RSFSR. Firmi!34». A un'eventuale richiesta di delucidazioni seguiva, nel migliore dei casi, una risposta negativa. L’ignoranza delle norme era dunque un elemento caratterizzante. Non per negligenza della popolazione, quanto perché non v'era possibilità alcuna di entrare in possesso del Codice, né quantomeno vederlo. Anche perché consultandolo ci si sarebbe potuti imbattere nell'articolo 136 del Codice di procedura penale che recitava: «Il giudice istruttore non ha il diritto di estorcere la deposizione di un imputato mediante violenze o minacce» 35. Ma tanto chi poteva saperlo? Quali violenze? Quali Gulag? Quali operazioni segrete? Non accadeva nulla di tutto ciò in Unione Sovietica. Le uniche purghe erano state quelle necessarie a ripulire il Partito, ed erano state compiute nel modo più legale $\mathrm{e}$ pubblico possibile, attraverso pubblici processi - su tutti quelli tenuti a Mosca - senza coercizione alcuna, rispettando il Codice, applicando norme scritte di fronte a rei confessi. Questa la superficie. La profondità era però altra cosa. E venne smascherata, in parte, dopo la morte di Stalin. Il famigerato rapporto segreto pronunciato da Nikita Chruščëv la notte tra il 24 e il 25 febbraio 1956 illuse però la stragrande maggioranza dei sopravvissuti e i parenti delle vittime della repressione stalinista. Il rapporto Chruščëv infatti evocava solamente le purghe dei dirigenti e dei quadri politici, economici e militari della cosiddetta nomenklatura, tenendo nascoste le operazioni di massa. Circoscrivendo il tutto ai soli dirigenti comunisti, vittime della dittatura personale di Stalin, si sollevava il Partito da ogni responsabilità. Partito che però era stato purgato a fondo ed era composto ormai dai soli dirigenti che avevano superato indenni il Terrore. Qui si evidenzia l'influenza delle purghe politiche stesse nella storia dell'URSS. A governare l'Unione Sovietica saranno, fino a Gorbačëv, dirigenti formati in quel periodo. Malenkov, Chruščëv, Brežnev, Černenko e Andropov, erano tutti personaggi politici formatisi alla fine degli anni Trenta, durante il repulisti del Partito. Molotov nel 1985 ancora sosteneva l'indispensabilità del Grande Terrore ${ }^{36}$, ritenendolo

\footnotetext{
34 Ibidem, p. 135.

35 Ibidem.

${ }^{36}$ Anche se non nominava la parola Terrore.
} 
passaggio essenziale per la costruzione della potenza sovietica. Forse aveva ragione: l'URSS senza Terrore non sarebbe stata l'URSS capace da sola di tener testa al mondo occidentale per mezzo secolo. Alla domanda «è servito il Terrore?» si dovrà rispondere, cinicamente, in maniera positiva. Su che cosa sia stato il Terrore si dovrà ricordare quanto scriveva Solcenizyn: «㐫 fatta, siete arrestato. E voi non troverete altro da rispondere che un belato da agnello: "I-io? Perché?»37.

${ }^{37}$ SOLZENICYN, Aleksandr, op. cit., p. 20. 


\section{* L'autore}

Filippo Ferrara ha conseguito la Laurea Triennale in Scienze della Comunicazione, indirizzo politico-istituzionale, presso l'Università degli studi di Roma Tor vergata, con una tesi sulla continuità e la rottura della politica staliniana rispetto i principi ispiranti la rivoluzione d'ottobre e la politica di Lenin. Ha collaborato con un quotidiano locale, a cadenza settimanale, scrivendo articoli di politica e di sport. Attualmente è iscritto alla Laurea Magistrale in Scienze storiche dell'Università di Bologna.

URL: < http://www.studistorici.com/progett/autori/\#Ferrara >

\section{Per citare questo articolo:}

FERRARA, Filippo, «Pulizia, giustizia e potere. Ruolo e significato del Terrore nell'esperienza staliniana», Diacronie. Studi di Storia Contemporanea : Processo penale, politica, opinione pubblica (secoli XVIII-XX), 29/08/2013,

URL:< http://www.studistorici.com/2013/08/29/ferrara_numero_14/ >

\section{Diacronie Studi di Storia Contemporanea $\beta$ www.diacronie.it}

Risorsa digitale indipendente a carattere storiografico. Uscita trimestrale.

redazione.diacronie@hotmail.it

Comitato di redazione: Marco Abram - Jacopo Bassi - Luca Bufarale - Alessandro Cattunar - Elisa Grandi - Deborah Paci - Fausto Pietrancosta - Matteo Tomasoni - Luca Zuccolo

Diritti: gli articoli di Diacronie. Studi di Storia Contemporanea sono pubblicati sotto licenza Creative Commons 2.5 Possono essere riprodotti a patto di non modificarne i contenuti e di non usarli per fini commerciali. La citazione di estratti è comunque sempre autorizzata, nei limiti previsti dalla legge. 